\title{
A HYBRID COLLOCATION METHOD FOR VOLTERRA INTEGRAL EQUATIONS WITH WEAKLY SINGULAR KERNELS*
}

\author{
YANZHAO $\mathrm{CAO}^{\dagger}$, TERRY HERDMAN ${ }^{\ddagger}$, AND YUESHENG XU $\mathrm{XU}^{\S}$
}

\begin{abstract}
The commonly used graded piecewise polynomial collocation method for weakly singular Volterra integral equations may cause serious round-off error problems due to its use of extremely nonuniform partitions and the sensitivity of such time-dependent equations to round-off errors. The singularity preserving (nonpolynomial) collocation method is known to have only local convergence. To overcome the shortcoming of these well-known methods, we introduce a hybrid collocation method for solving Volterra integral equations of the second kind with weakly singular kernels. In this hybrid method we combine a singularity preserving (nonpolynomial) collocation method used near the singular point of the derivative of the solution and a graded piecewise polynomial collocation method used for the rest of the domain. We prove the optimal order of global convergence for this method. The convergence analysis of this method is based on a singularity expansion of the exact solution of the equations. We prove that the solutions of such equations can be decomposed into two parts, with one part being a linear combination of some known singular functions which reflect the singularity of the solutions and the other part being a smooth function. A numerical example is presented to demonstrate the effectiveness of the proposed method and to compare it to the graded collocation method.
\end{abstract}

Key words. Volterra integral equations, hybrid collocation methods, weakly singular kernels

AMS subject classifications. 65R20, 45D05

\section{PII. S0036142901385593}

1. Introduction. We propose in this paper a hybrid collocation method for solving Volterra integral equations of the second kind with weakly singular kernels. By using the singularity expansion of the exact solution, we analyze this method and prove that it has an optimal order of global convergence. Specifically, for given kernels $K, M \in C(I \times I)$ with $I:=[0,1]$ and a given parameter $\alpha \in(0,1)$, we define a Volterra integral operator $T_{\alpha}: C(I) \rightarrow C(I)$ by

$$
\left(T_{\alpha} y\right)(t)=\int_{0}^{t} G_{\alpha}(t, s) y(s) d s, \quad t \in I,
$$

where

$$
G_{\alpha}(t, s):=(t-s)^{\alpha-1} K(t, s)+M(t, s) \text { for } 0 \leq s \leq t, \quad 0 \leq t \leq 1,
$$

and consider the Volterra integral equation of the second kind

$$
y(t)-\left(T_{\alpha} y\right)(t)=f(t), \quad t \in I,
$$

* Received by the editors February 26, 2001; accepted for publication (in revised form) October 14, 2002; published electronically April 9, 2003.

http://www.siam.org/journals/sinum/41-1/38559.html

†Department of Mathematics, Florida A \& M University, Tallahassee, FL 32307 (yanzhao.cao@ mail.famu.edu).

${ }_{\ddagger}^{\ddagger}$ Department of Mathematics, Virginia Tech, Blacksburg, VA 24061 (herdman@math.vt.edu).

$\S$ Corresponding author. Department of Mathematics, West Virginia University, Morgantown, WV 26506 and Academy of Mathematics and System Sciences, Academia Sinica, Beijing 100080, China (yxu@math.wvu.edu). This author was supported in part by the National Science Foundation under grant DMS-9973427 and by the Chinese Academy of Sciences under the program of "One Hundred Distinguished Young Scientists." 
where $f \in C(I)$ is a given function and $y \in C(I)$ is the unknown function to be determined. The kernel $M$ is of practical importance because it occurs in the applications to aeroelastic modeling problems [7], where a class of neutral delay equations are converted to integral equations in the form of (1.1). Other related references include $[4,11]$.

Since $0<\alpha<1$, the kernel $G_{\alpha}$ has a singularity along the diagonal. When (1.1) is solved by a numerical method such as a collocation method or a productintegration method using the piecewise polynomial approximation, the accuracy of the approximate solution depends on the order of piecewise polynomials used in the approximation as well as the degree of smoothness of the exact solution. For instance, when $y \in C^{r}(I)$ and the approximate subspaces are chosen to be piecewise polynomials of order $r$, the optimal order $r$ of convergence for the approximate solution $y_{h}$ to $y$ is achieved, that is,

$$
\left\|y-y_{h}\right\|_{\infty}=O\left(N^{-r}\right)
$$

where $N$ is the number of subintervals in the uniform partition associated with the piecewise polynomial spaces. However, the solution of (1.1) exhibits, in general, singularities at the zero in its derivatives even if the forcing term $f$ is a smooth function and the numerical methods mentioned above may not even yield first order accuracy (see, e.g., $[2,5]$ ). In other words, the use of piecewise polynomials of high order does not produce high order convergence for the numerical method.

There have been many attempts to overcome the difficulties caused by the singularity of the solution of (1.1). One of the most commonly used methods $[3,5,6$, $9,10,14,15,16]$ is the graded collocation (GC) method using piecewise polynomials with a graded mesh on interval $I$ according to the behavior of the exact solution near the singular point, which was first introduced by Rice in [13]. Specifically, the GC method partitions $I$ by the following knots:

$$
t_{i}=\left(\frac{i}{N}\right)^{\frac{r}{\alpha}}, \quad i=0,1, \ldots, N,
$$

which ensures that the GC method retains the optimal error estimate (1.2). However, as pointed out in $[2,10]$, the main disadvantage of the GC method is that subintervals near the singular point in the graded mesh have very small length and thus may cause serious round-off error problems for small $\alpha$ and high order polynomials. Since Volterra equations are time-dependent equations, the numerical solutions of these equations are very sensitive to round-off errors.

Another approach for solving (1.1) is to include some nonpolynomial singular functions which reflect the singularity of the exact solution as part of the basis for the finite dimensional subspace in the collocation method (see [2]). We call it the nonpolynomial collocation (NPC) method. For this method, only a local convergence result (in [2]) has been seen so far. It does not seem that an optimal order of global convergence can be proved for this method. The idea of including some known singular functions in the usual finite element spaces or piecewise polynomial spaces has been explored in [8] to successfully construct Galerkin methods of high convergence order for Fredholm integral equations of the second kind with weakly singular kernels. This idea leads us to the consideration of the present method.

To treat the problems discussed above for the existing methods, we propose a hybrid collocation (HC) method for solving (1.1) which combines the strength of both the GC and NPC methods. In this method, we introduce a graded mesh different from 
(1.3) that avoids using small subintervals near the zero and uses the nonpolynomial function approximation only in the first subinterval. Specifically, the length of the first subinterval in the HC method is the same as in a quasi-uniform partition, that is, there exist positive constants $c_{1}, c_{2}$ such that

$$
\frac{c_{1}}{N} \leq t_{1} \leq \frac{c_{2}}{N}
$$

and a graded partition is used only on $\left[t_{1}, 1\right]$ so that the instability problem appearing in the GC method can be avoided. We compensate the use of a large subinterval for the first interval in the partition by employing nonpolynomial functions $t^{i+j \alpha}, i+j \alpha<r$, which characterize the singularity of the exact solution $y$ of (1.1), as trial functions in the first subinterval $\left[t_{0}, t_{1}\right]$. The primary purpose of this paper is to prove that this method provides an optimal order of global convergence by taking the strength of both the GC method and the NPC method, while avoiding the problems from which both these methods have suffered.

To prepare for the analysis of this method, we derive a singularity expansion of the exact solution of (1.1). In other words, we decompose the exact solution into two parts, one being a linear combination of singular functions $t^{i+j \alpha}$ which reflect the singularity of the exact solution and the other being a smooth function. This subject has been well studied in [5]. We will make use of the results presented in [5] and construct further a form of expansion that is useful for the development of the $\mathrm{HC}$ method.

We organize this paper in five sections. In section 2 , we derive the singularity expansion of the exact solution of (1.1). Section 3 is devoted to a study of hybrid interpolation operators which serve as a base for the development of the HC method. In section 4, we describe the HC method which combines the NPC method used near the singular point based on the singularity expansion obtained in section 2 and a GC method elsewhere. We prove the optimal order of global convergence of this method. Furthermore, we present a theoretical result which gives a comparison of the computational cost of the HC method and the GC method, and the length of the smallest subintervals used in both methods. Our theory shows that the HC method is better than the GC method. Finally in section 5, we provide a numerical example to demonstrate the effectiveness of the $\mathrm{HC}$ method. We compare the numerical performance of the HC method with that of the GC method. The numerical results confirm the theory presented in section 4 .

2. Singularity expansions. In this section we establish a preliminary result on the singularity decomposition for the solution of (1.1). Singularity of the solution of (1.1) when the kernel $M$ is zero has been systematically studied in [5]. In the next theorem, we make use of the results in [5] and derive the singularity expansion crucial for the development of the $\mathrm{HC}$ method for the general case when $M \neq 0$.

Theorem 2.1. Let $r$ be a nonnegative integer. Suppose that $K, M \in C^{r}(I \times I)$ and $f$ has the form

$$
f(t)=\sum_{j+i \alpha<m} f_{i j} t^{j+i \alpha}+f_{m}(t), \quad t \in I,
$$

where $f_{i j}$ are constants and $f_{m} \in C^{m}[0,1]$ for some fixed integer $m$ with $0 \leq m \leq r$. Let $y$ denote the solution of (1.1). Then there exist constants $c_{i j}$ such that

$$
y(t)=\sum_{j+i \alpha<m} c_{i j} t^{j+i \alpha}+v_{m}(t), \quad t \in I
$$


where $v_{m} \in C^{m}(I)$.

Proof. When $M \equiv 0$ formula (2.2) follows from Theorem 1.3.15 of [5] with some modification. The modification is necessary to treat the series in the expansion appearing in Theorem 1.3.15 of [5] so that we have form (2.2).

The general case where the kernel $M$ is not zero will be proved by induction on $m$. The case when $m=0$ is obvious. We assume that the theorem holds for $m=k$ and proceed to the case $m=k+1$. By the induction hypothesis, the solution $y$ of (1.1) has a representation

$$
y(t)=\sum_{j+i \alpha<k} c_{i j} t^{j+i \alpha}+v_{k}(t), \quad t \in I,
$$

where $v_{k} \in C^{k}(I)$. For $t \in I$, we let

$$
x(t):=\int_{0}^{t} M(t, s) y(s) d s .
$$

Substituting (2.3) into the expression of function $x$ gives

$$
x(t)=\int_{0}^{t} M(t, s)\left[\sum_{j+i \alpha<k} c_{i j} s^{j+i \alpha}+v_{k}(s)\right] d s, \quad t \in I .
$$

To simplify the expression of $x$, we denote

$$
w_{k+1}(t)=\int_{0}^{t} M(t, s) v_{k}(s) d s \text { and } m_{i j}(t)=\int_{0}^{1} M(t, s t) s^{j+i \alpha} d s .
$$

A simplification with a change of variables leads to the following formula:

$$
x(t)=\sum_{j+i \alpha<k+1} c_{i, j-1} m_{i, j-1}(t) t^{j+i \alpha}+w_{k+1}(t), \quad t \in I .
$$

It is easily seen that $w_{k+1}, m_{i j} \in C^{k+1}(I)$. Applying the Taylor theorem to the functions $m_{i j}$, we obtain that

$$
x(t)=\sum_{j+i \alpha<k+1} d_{i j} t^{j+i \alpha}+u_{k+1}(t), \quad t \in I,
$$

where $d_{i j}$ are constants and $u_{k+1}$ is a function in $C^{k+1}(I)$. Let $\tilde{f}:=f-x$ and rewrite (1.1) as

$$
y(t)+\int_{0}^{t}(t-s)^{\alpha-1} K(t, s) y(s) d s=\tilde{f}(t), \quad t \in I .
$$

Note that $\tilde{f}$ has the form (2.1) with $m=k+1$. By the first part of this proof, we conclude the result of the theorem for the case $m=k+1$, which advances the induction hypothesis and completes the proof. 
3. Nonpolynomial interpolation operators. Motivated by the singularity expansion of the solution of (1.1), in the next section we will develop the HC method for solving (1.1). To prepare for this development, we define a hybrid interpolation operator and study the bound of this operator.

We first define a nonpolynomial finite dimensional subspaces of $C(I)$. As usual, we denote by $N_{0}$ the set of nonnegative integers. For $0<\alpha<1$ and a positive integer $r$ we introduce an index set by setting

$$
W_{\alpha, r}:=\left\{i+j \alpha: i, j \in N_{0}, i+j \alpha<r\right\} .
$$

Let $\ell$ denote the cardinality of the set $W_{\alpha, r}$. Clearly, $W_{\alpha, r}$ contains the first $r$ nonnegative integers $i=0,1, \ldots, r-1$. For notational convenience, we write

$$
W_{\alpha, r}=\left\{\nu_{j}: j=0,1, \ldots, \ell-1\right\}
$$

with the convention that $\nu_{j}=j$ for $j=0,1, \ldots, r-1$. Associated with this index set, we define a finite dimensional space $V_{r}$ of nonpolynomial functions by

$$
V_{r}:=\operatorname{span}\left\{t^{\nu_{j}}: j=0,1, \ldots, \ell-1\right\}
$$

We remark that Theorem 2.1 ensures the solution $y$ of (1.1) has the decomposition

$$
y=u+v, \quad u \in C^{r}(I), \text { and } v \in V_{r} .
$$

Also, we denote by $P_{r}$ the space of polynomials of degree $\leq r-1$. Because the set $W_{\alpha, r}$ contains the integers $i=0,1, \ldots, r-1$, it is clear that $P_{r} \subset V_{r}$. In addition, space $V_{r}$ contains nonpolynomial functions $t^{\nu_{j}}, j=r, r+1, \ldots, \ell-1$, that reflect the singularity of the derivative of the solution of (1.1).

We next describe a finite dimensional space whose elements are piecewise in $V_{r}$. For a given positive integer $N$, we divide the interval $I$ into $N$ subintervals, that is, $0=t_{0}<t_{1}<\cdots<t_{N}=1$. For a subinterval $J$ of $I$ and a function $f \in C(I)$, we use $\left.f\right|_{J}$ for the restriction of $f$ on $J$ and, moreover, for $V \subseteq C(I)$ we let

$$
\left.V\right|_{J}:=\left\{\left.v\right|_{J}: v \in V\right\}
$$

Let $h_{i}=t_{i}-t_{i-1}$ and $h=\max _{1 \leq i \leq N} h_{i}$. We define a space of functions piecewise in $V$ by

$$
(V)_{h}:=\left\{v:\left.\left.v\right|_{\left[t_{i-1}, t_{i}\right]} \in V\right|_{\left[t_{i-1}, t_{i}\right]}, i=1,2, \ldots, N\right\},
$$

and, in particular, we let $V_{r, h}:=\left(V_{r}\right)_{h}$ and $S_{r, h}:=\left(P_{r}\right)_{h}$. Clearly, we have that $V_{r} \subseteq V_{r, h}$ and $S_{r, h} \subseteq V_{r, h}$.

We now define interpolation operators $P_{h, 1}$ from $C(I)$ to $S_{r, h}$ and $P_{h, 2}$ from $C(I)$ to $V_{r, h}$, respectively. To this end, we choose $\ell$ points $\tau_{j}$ in $I$ such that $0<\tau_{1}<\tau_{2}<$ $\cdots<\tau_{\ell}<1$. The interpolation points on interval $\left[t_{k-1}, t_{k}\right]$ are obtained by setting $t_{k j}:=t_{k-1}+\tau_{j} h_{k}, k=1,2, \ldots, N, j=1,2, \ldots, \ell$. The interpolation operators $P_{h, 1}: C(I) \rightarrow S_{r, h}$ and $P_{h, 2}: C(I) \rightarrow V_{r, h}$ are defined as follows. For $f \in C(I)$

$$
\left(P_{h, 1} f\right)\left(t_{i j}\right)=f\left(t_{i j}\right), \quad j=1,2, \ldots, r, i=1,2, \ldots, N,
$$

and

$$
\left(P_{h, 2} f\right)\left(t_{i j}\right)=f\left(t_{i j}\right), \quad j=1,2, \ldots, \ell, i=1,2, \ldots, N .
$$


Note that for the definition of $P_{h, 1}$ we use only the first $r$ points $\tau_{j}, j=1,2, \ldots, r$. It is known (see $[2,5]$ ) that the operators $P_{h, i}$ for $i=1,2$ are uniquely defined.

For a function $u$ that is continuous on $\left[t_{i-1}, t_{i}\right], i=1,2, \ldots, N$, with possible discontinuities at $t_{i}$, define its maximum norm on $\left[t_{m}, t_{n}\right]$ with $0 \leq m<n \leq N$ by

$$
\|u\|_{\left[t_{m}, t_{n}\right]}=\max _{m \leq i \leq n} \max _{t_{i-1} \leq t \leq t_{i}}|u(t)| .
$$

We will simply use $\|u\|$ when $\left[t_{m}, t_{n}\right]=I$.

Next we show that the norm of the restriction of $P_{h, 2} f$ on $\left[t_{0}, t_{1}\right]$ is bounded by a constant independent of the choice of $t_{1}$. For this purpose, we define, for $i=1,2, \ldots, \ell$, the Lagrange functions

$$
\left.\left.L_{1 i}\right|_{\left[t_{0}, t_{1}\right]} \in V_{r}\right|_{\left[t_{0}, t_{1}\right]}
$$

and

$$
L_{1 i}(t)=0, \quad t \in\left[t_{1}, 1\right]
$$

such that

$$
L_{1 i}\left(t_{1 j}\right)=\delta_{i j}, \quad j=1,2, \ldots, \ell
$$

It is easily verified that

$$
\left(P_{h, 2} f\right)(t)=\sum_{j=1}^{\ell} f\left(t_{1 j}\right) L_{1 j}(t), \quad t \in\left[t_{0}, t_{1}\right] .
$$

Lemma 3.1. There exists a positive constant $c$ such that for all $t_{1} \in(0,1)$ and $i=1,2, \ldots, \ell$

$$
\left\|L_{1 i}\right\|=\left\|L_{1 i}\right\|_{\left[t_{0}, t_{1}\right]} \leq c .
$$

Proof. For $i=1,2, \ldots, \ell$, we write

$$
L_{1 i}(t)=\sum_{p=1}^{\ell} a_{i p} t^{\nu_{p}}, \quad t \in\left[t_{0}, t_{1}\right] .
$$

For $t \in\left[t_{0}, t_{1}\right]$, there exists a $\tau \in I$ such that $t=t_{0}+h_{1} \tau$. For $i=1,2, \ldots, \ell$ and $p=1,2, \ldots, \ell$ we set $b_{i p}:=h_{1}^{\nu_{p}} a_{i p}$. Using these notations, we have that

$$
L_{1 i}(t)=\sum_{p=1}^{\ell} b_{i p} \tau^{\nu_{p}}, \quad t \in\left[t_{0}, t_{1}\right] .
$$

By the definition of $L_{1 i}$ we observe that

$$
\sum_{p=1}^{\ell} b_{i p} \tau_{j}^{\nu_{p}}=\delta_{i j}, \quad j=1,2, \ldots, \ell .
$$

We introduce an $\ell \times \ell$ matrix $\mathbf{D}$ by setting $\mathbf{D}:=\left[d_{j p}: j, p=1,2, \ldots, \ell\right]$, where $d_{j p}:=\tau_{j}^{\nu_{p}}$ and a vector $\mathbf{b}_{i}$ by $\mathbf{b}_{i}=\left[b_{i p}: p=1,2, \ldots, \ell\right]^{T}$. It is easy to verify that the matrix $\mathbf{D}$ is invertible and since all entries $d_{j p}$ are independent of the choice of $t_{1}$ 
we conclude that $\left\|\mathbf{D}^{-1}\right\|_{\infty}$ is bounded by a constant independent of the choice of $t_{1}$ where the norm used here is the matrix norm induced from the vector norm $\|\cdot\|_{\infty}$. Thus, it follows from (3.3) that

$$
\left\|\mathbf{b}_{i}\right\|_{\infty} \leq\left\|\mathbf{D}^{-1}\right\|_{\infty}
$$

Hence from (3.2) we confirm the result of this lemma with the constant

$$
c:=\max _{0 \leq \tau \leq 1}\left(\sum_{p=1}^{\ell} \tau^{\nu_{p}}\right)\left\|\mathbf{D}^{-1}\right\|_{\infty} .
$$

The following lemma, which provides a bound of the norm of $P_{h, 2} f$ on $\left[t_{0}, t_{1}\right]$, is a direct consequence of Lemma 3.1 .

Lemma 3.2. There exists a positive constant $c$ such that for all $t_{1} \in(0,1)$ and for any $f \in C(I)$

$$
\left\|P_{h, 2} f\right\|_{\left[t_{0}, t_{1}\right]} \leq c\|f\|_{\left[t_{0}, t_{1}\right]} .
$$

The next proposition presents order of convergence for the interpolation $P_{h, 2} f$ to a function $f$ having form (2.1).

Proposition 3.3. There exists a positive constant $c$ such that for all $t_{1} \in(0,1)$ and for $f=u+v$, where $u \in C^{r}(I)$ and $v \in V_{r}$,

$$
\left\|f-P_{h, 2} f\right\|_{\left[t_{0}, t_{1}\right]} \leq c h_{1}^{r}\left\|u^{(r)}\right\|_{\left[t_{0}, t_{1}\right]} .
$$

Proof. For all functions $f$ having the form $f=u+v$, where $u \in C^{r}(I)$ and $v \in V_{r}$, recalling that $P_{h, 2} v=v$ for $v \in V_{r}$ we have that

$$
f-P_{h, 2} f=u+v-P_{h, 2}(u+v)=u-P_{h, 2} u .
$$

Noting that

$$
P_{h, 2} P_{h, 1}=P_{h, 1}
$$

we conclude that

$$
f-P_{h, 2} f=\left(I-P_{h, 1}\right) u+P_{h, 2} P_{h, 1} u-P_{h, 2} u=\left(I-P_{h, 2}\right)\left(I-P_{h, 1}\right) u .
$$

It follows from Lemma 3.2 that there exists a positive constant $c$ such that for all $t_{1} \in(0,1)$ and all such $f$

$$
\left\|f-P_{h, 2} f\right\|_{\left[t_{0}, t_{1}\right]} \leq c\left\|\left(I-P_{h, 1}\right) u\right\|_{\left[t_{0}, t_{1}\right]} \leq c h_{1}^{r}\left\|u^{(r)}\right\|_{\left[t_{0}, t_{1}\right]},
$$

where the last inequality follows from a standard error estimate for polynomial interpolations.

We next define a hybrid interpolation which has a global convergence. To this end, we describe a graded partition of $I$ in terms of parameters $\alpha$ and $r$. Specifically, for $q:=\frac{r}{\alpha}$ we let $i_{0}$ be an integer such that

$$
\left[\left(\frac{N}{i_{0}}\right)^{q}\right]=N
$$


where $[a]$ denotes the largest integer less than or equal to $a$, and set $N^{\prime}:=N-i_{0}+1$. The partition on $I$ is given by

$$
t_{0}=0, t_{i}=\left(\frac{i_{0}+i-1}{N}\right)^{q}, \quad i=1,2, \ldots, N^{\prime} .
$$

Note that $t_{N^{\prime}}=1$ and the integer $i_{0}$ satisfies the condition that

$$
N^{1-1 / q} \leq i_{0} \leq N(N-1)^{-1 / q} .
$$

We remark that as far as stability is concerned this partition is better than the partition (1.3) used in a standard GC method. This point will be made clearer in the next section.

Associated with the graded partition (3.4), we define the hybrid interpolation operator $Q_{h}$ by

$$
\left.\left(Q_{h} f\right)\right|_{\left[0, t_{1}\right]}=\left.\left(P_{h, 2} f\right)\right|_{\left[0, t_{1}\right]} \text { and }\left.\left(Q_{h} f\right)\right|_{\left[t_{1}, 1\right]}=\left.\left(P_{h, 1} f\right)\right|_{\left[t_{1}, 1\right]} .
$$

That is, on the first subinterval we use singularity preserving (nonpolynomial) interpolation and on the rest of intervals we use the standard piecewise polynomial interpolation. The operator $Q_{h}$ will be used in the next section for the development of a hybrid collocation method. To prepare for this development, we present an expression of projection $Q_{h}$ in terms of the Lagrange basis functions. To this end, we define the Lagrange polynomial basis $L_{i} \in P_{r}, i=1,2, \ldots, r$, such that

$$
L_{i}\left(\tau_{j}\right)=\delta_{i j}, \quad j=1,2, \ldots, r,
$$

and for $k=2,3, \ldots, N^{\prime}$ we define the Lagrange piecewise polynomial basis functions by setting

$$
L_{k i}(t)= \begin{cases}L_{i}\left(\frac{t-t_{k-1}}{h_{k}}\right), & t \in\left[t_{k-1}, t_{k}\right], \\ 0, & \text { otherwise }\end{cases}
$$

Thus, for all $k=2,3 \ldots, N^{\prime}$ there hold the relations that

$$
\left\|L_{k i}\right\|=\left\|L_{i}\right\|, \quad i=1,2, \ldots, r
$$

and we have that

$$
\left(P_{h, 1} f\right)(t)=\sum_{k=2}^{N^{\prime}} \sum_{j=1}^{r} f\left(t_{k j}\right) L_{k j}(t), \quad t \in\left[t_{1}, 1\right] .
$$

To present projection $Q_{h}$, we introduce a notation

$$
r_{k}:= \begin{cases}\ell, & k=1 \\ r, & k=2,3, \ldots, N^{\prime} .\end{cases}
$$

Consequently, we have that

$$
\left(Q_{h} f\right)(t)=\sum_{k=1}^{N^{\prime}} \sum_{j=1}^{r_{k}} f\left(t_{k j}\right) L_{k j}(t), \quad t \in I .
$$


In the next proposition, we establish a global convergence result for the interpolation projection $Q_{h}$.

Proposition 3.4. Let $Q_{h}$ be the hybrid interpolation operator defined in (3.6) associated with the partition (3.4). Suppose that $f$ has a decomposition $f=u+v$, where $u \in C^{r}(I)$ and $v \in V_{r}$. Then there exists a positive constant $c$ independent of $N$ such that for all such functions $f$

$$
\left\|f-Q_{h} f\right\| \leq c N^{-r} .
$$

Proof. Note that

$$
\left\|f-Q_{h} f\right\|=\max \left\{\left\|f-Q_{h} f\right\|_{\left[0, t_{1}\right]},\left\|f-Q_{h} f\right\|_{\left[t_{1}, 1\right]}\right\} .
$$

Employing Proposition 3.3 we conclude that there exists a positive constant $c$ such that

$$
\left\|f-Q_{h} f\right\|_{\left[0, t_{1}\right]}=\left\|f-P_{h, 2} f\right\|_{\left[0, t_{1}\right]} \leq c h_{1}^{r}\left\|u^{(r)}\right\|_{\left[0, t_{1}\right]} .
$$

Noting that by the definition of partition (3.4),

$$
h_{1}=t_{1}=\left(\frac{i_{0}}{N}\right)^{q} \leq\left(\frac{N(N-1)^{-1 / q}}{N}\right)^{q}=\frac{1}{N-1} \leq \frac{2}{N}
$$

we obtain that

$$
\left\|f-Q_{h} f\right\|_{\left[0, t_{1}\right]} \leq c \frac{1}{N^{r}} .
$$

We next estimate the error $\left\|f-Q_{h} f\right\|_{\left[t_{1}, 1\right]}$ following a well-known argument by Rice [13]. Since $f=u+v$ with $u \in C^{r}(I)$ and $v \in V_{r}$, we have that

$$
\left|f^{(r)}(t)\right| \leq c t^{\alpha-r} \text { for } t \in\left[t_{1}, 1\right],
$$

and the function $t^{\alpha-r}$ is decreasing in $t$. For $i=2,3, \ldots, N^{\prime}$, we find that

$h_{i}=\left[\left(i+i_{0}-1\right)^{q}-\left(i+i_{0}-2\right)^{q}\right] N^{-q}=\left(i+i_{0}-2\right)^{q}\left\{\left[1+\left(i+i_{0}-2\right)^{-1}\right]^{q}-1\right\} N^{-q}$.

By the mean value theorem, there exists $\theta$ with $0<\theta<\left(i+i_{0}-2\right)^{-1}$ such that

$$
h_{i}=q\left(i+i_{0}-2\right)^{q-1}(1+\theta)^{q-1} N^{-q} \leq c\left(i+i_{0}-2\right)^{q-1} N^{-q} .
$$

Thus, there exists a positive constant $c$ such that for $i=2,3, \ldots, N^{\prime}$

$$
\begin{aligned}
\left\|f-Q_{h} f\right\|_{\left[t_{i-1}, t_{i}\right]} & =\left\|f-P_{h, 2} f\right\|_{\left[t_{i-1}, t_{i}\right]} \leq c h_{i}^{r}\left\|f^{(r)}\right\|_{\left[t_{i-1}, t_{i}\right]} \\
& \leq c\left(i+i_{0}-2\right)^{(q-1) r} N^{-q r}\left(\frac{i+i_{0}-2}{N}\right)^{q(\alpha-r)}=c \frac{1}{N^{r}},
\end{aligned}
$$

which completes the proof of this proposition.

4. A hybrid collocation method. In this section, we use the hybrid interpolation operator $Q_{h}$ introduced in the last section to develop a hybrid collocation method for solving (1.1). We prove that this method has an optimal order of global convergence. Notice that the singularity in the derivative of the exact solution of (1.1) occurs only at the left end point of the interval $I$. This fact suggests that we use a 
singularity preserving collocation method near the left end point and use a standard piecewise polynomial collocation method with a graded partition elsewhere.

We now describe the hybrid collocation method for (1.1). We seek $y_{h}$ such that

$$
\left.\left.y_{h}\right|_{\left[0, t_{1}\right]} \in V_{r, h}\right|_{\left[0, t_{1}\right]},\left.\left.\quad y_{h}\right|_{\left[t_{1}, 1\right]} \in S_{r, h}\right|_{\left[t_{1}, 1\right]},
$$

and

$$
y_{h}-Q_{h} T_{\alpha} y_{h}=Q_{h} f
$$

where $Q_{h}$ is the hybrid interpolation operator defined by (3.6) associated with the graded partition (3.4).

To analyze the order of convergence for the hybrid collocation method (4.1), we define an integral operator $T_{\alpha, 1}$ by

$$
\left(T_{\alpha, 1} y\right)(t):=\left(T_{\alpha} y\right)(t) \text { for } t \in\left[0, t_{1}\right]
$$

and

$$
\left(T_{\alpha, 1} y\right)(t):=\int_{t_{1}}^{t} G_{\alpha}(t, s) y(s) d s \text { for } t \in\left[t_{1}, 1\right] .
$$

The study of the error $\left\|y-y_{h}\right\|$ demands a bound on the errors

$$
e_{k j}:=y\left(t_{k j}\right)-y_{h}\left(t_{k j}\right), \quad j=1,2, \ldots, r_{k},
$$

where $r_{k}$ is defined by (3.8). To this end, we introduce vectors

$$
\mathbf{e}_{k}:=\left[e_{k j}: j=1,2, \ldots, r_{k}\right]^{T} \text { for } k=1,2, \ldots, N^{\prime} .
$$

We also need vectors

$$
\epsilon_{k}:=\left[\epsilon_{k j}: j=1,2, \ldots, r_{k}\right]^{T} \text { for } k=1,2, \ldots, N^{\prime},
$$

where

$$
\epsilon_{k j}:=\left(T_{\alpha, 1}\left(y-Q_{h} y\right)\right)\left(t_{k j}\right)+\left(\left(T_{\alpha}-T_{\alpha, 1}\right)\left(y-y_{h}\right)\right)\left(t_{k j}\right) .
$$

Note that when $k=1$, it becomes that

$$
\epsilon_{1 j}=\left(T_{\alpha}\left(y-Q_{h} y\right)\right)\left(t_{1 j}\right) .
$$

We will bound the vectors $\mathbf{e}_{k}$ by $\epsilon_{k}$.

Next, we derive a linear system that gives a recursive formula for the vector $\mathbf{e}_{k}$. Toward this goal, for $k=1,2, \ldots, N^{\prime}$ and $j=1,2, \ldots, r_{k}$ we evaluate (1.1) and (4.1) at $t_{k j}$ to obtain that

$$
y\left(t_{k j}\right)-\left(T_{\alpha} y\right)\left(t_{k j}\right)=f\left(t_{k j}\right)
$$

and

$$
y_{h}\left(t_{k j}\right)-\left(T_{\alpha} y_{h}\right)\left(t_{k j}\right)=f\left(t_{k j}\right),
$$

respectively. Subtracting (4.5) from (4.4) yields

$$
e_{k j}=\left(T_{\alpha, 1}\left(y-y_{h}\right)\right)\left(t_{k j}\right)+\left(\left(T_{\alpha}-T_{\alpha, 1}\right)\left(y-y_{h}\right)\right)\left(t_{k j}\right) .
$$


Noticing that $Q_{h}$ is a projection and $y_{h}=Q_{h} y_{h}$, we have that

$$
y-y_{h}=y-Q_{h} y+Q_{h}\left(y-y_{h}\right) .
$$

Substituting (4.7) into the first term in the right-hand side of (4.6) and recalling the definition of $\epsilon_{k j}$, we obtain that

$$
e_{k j}=\left(T_{\alpha, 1} Q_{h}\left(y-y_{h}\right)\right)\left(t_{k j}\right)+\epsilon_{k j} \text { for } k=1,2, \ldots, N^{\prime}, j=1,2, \ldots, r_{k} .
$$

We are required to study the first term in the right-hand side of (4.8). In (3.9), we replace $f$ by $y-y_{h}$ and conclude that

$$
\left(Q_{h}\left(y-y_{h}\right)\right)(t)=\sum_{i=1}^{N^{\prime}} \sum_{p=1}^{r_{i}} e_{i p} L_{i p}(t), \quad t \in I .
$$

Applying the operator $T_{\alpha, 1}$ to both sides of this equation with evaluating at $t=t_{k j}$ yields

$$
\left(T_{\alpha, 1} Q_{h}\left(y-y_{h}\right)\right)\left(t_{k j}\right)=\sum_{i=1}^{N^{\prime}} \sum_{p=1}^{r_{i}} e_{i p}\left(T_{\alpha, 1} L_{i p}\right)\left(t_{k j}\right) .
$$

We next make use of the property of the Lagrange basis functions $L_{i p}$ to simplify the right-hand side of the equation above. For notational convenience, we define

$$
a_{j p}^{k}:=\int_{t_{k-1}}^{t_{k j}} G_{\alpha}\left(t_{k j}, s\right) L_{k p}(s) d s
$$

and

$$
d_{j p}^{k i}:=\int_{t_{i-1}}^{t_{i}} G_{\alpha}\left(t_{k j}, s\right) L_{i p}(s) d s
$$

Noting that $L_{i p}$ vanishes outside the interval $\left[t_{i-1}, t_{i}\right]$, an elementary computation leads to the formula that

$$
\left(T_{\alpha, 1} Q_{h}\left(y-y_{h}\right)\right)\left(t_{k j}\right)=\sum_{p=1}^{r_{k}} a_{j p}^{k} e_{k p}+\sum_{i=2}^{k-1} \sum_{p=1}^{r} d_{j p}^{k i} e_{i p},
$$

where we have used the relation that $r_{i}=r$ for $i=2,3, \ldots, k-1$. Substituting this equation into the right-hand side of (4.8) yields

$$
e_{k j}=\sum_{p=1}^{r_{k}} a_{j p}^{k} e_{k p}+\sum_{i=2}^{k-1} \sum_{p=1}^{r} d_{j p}^{k i} e_{i p}+\epsilon_{k j}, \quad j=1,2, \ldots, r_{k} .
$$

By introducing an $r_{k} \times r_{k}$ matrix

$$
\mathbf{A}_{k}:=\left[a_{j p}^{k}: j, p=1,2, \ldots, r_{k}\right]
$$

and $r \times r$ matrices

$$
\mathbf{D}_{k i}:=\left[d_{j p}^{k i}: j, p=1,2, \ldots, r\right], \quad i=2,3, \ldots, k-1,
$$


we write (4.9) in matrix form as

$$
\mathbf{e}_{k}=\mathbf{A}_{k} \mathbf{e}_{k}+\sum_{i=2}^{k-1} \mathbf{D}_{k i} \mathbf{e}_{i}+\epsilon_{k}, \quad k=1,2, \ldots, N^{\prime} .
$$

The matrices $\mathbf{A}_{k}$ and $\mathbf{D}_{k i}$ are all dependent on the mesh sizes $h_{i}$, and we next study such a dependence. Recalling the transformations $s=t_{k-1}+h_{k} \tau$ when $s \in$ $\left[t_{k-1}, t_{k}\right]$ and $\tau \in[0,1]$ and $t_{k j}=t_{k-1}+h_{k} \tau_{j}$ and using the notations

$$
\begin{gathered}
\tilde{K}(t, \tau):=K\left(t, t_{k-1}+h_{k} \tau\right), \tilde{M}(t, \tau):=M\left(t, t_{k-1}+h_{k} \tau\right), \tilde{L}_{k p}(\tau):=L_{k p}\left(t_{k-1}+h_{k} \tau\right), \\
\tilde{a}_{j p}^{k}:=\int_{0}^{\tau_{j}}\left[\left(\tau_{j}-\tau\right)^{\alpha-1} \tilde{K}\left(t_{k j}, \tau\right)+h_{k}^{1-\alpha} \tilde{M}\left(t_{k j}, \tau\right)\right] \tilde{L}_{k p}(\tau) d \tau,
\end{gathered}
$$

and

$$
\tilde{d}_{j p}^{k i}:=\int_{0}^{1}\left[\left(\frac{t_{k-1}-t_{i-1}+h_{k} \tau_{j}}{h_{i}}-\tau\right)^{\alpha-1} \tilde{K}\left(t_{k j}, \tau\right)+h_{i}^{1-\alpha} \tilde{M}\left(t_{k j}, \tau\right)\right] \tilde{L}_{i p}(\tau) d \tau,
$$

by changes of variables we have that

$$
a_{j p}^{k}=h_{k}^{\alpha} \tilde{a}_{j p}^{k} \text { and } d_{j p}^{k i}=h_{i}^{\alpha} \tilde{d}_{j p}^{k i}
$$

By introducing new matrices

$\tilde{\mathbf{A}}_{k}:=\left[\tilde{a}_{j p}^{k}: j, p=1,2, \ldots, r_{k}\right]$ and $\tilde{\mathbf{D}}_{k i}:=\left[\tilde{d}_{j p}^{k i}: j, p=1,2, \ldots, r\right], \quad i=2,3, \ldots, k-1$, we observe that

$$
\tilde{\mathbf{A}}_{k}=h_{k}^{-\alpha} \mathbf{A}_{k} \text { and } \tilde{\mathbf{D}}_{k i}=h_{k}^{-\alpha} \mathbf{D}_{k i}
$$

Hence, (4.12) becomes

$$
\mathbf{e}_{k}=h_{k}^{\alpha} \tilde{\mathbf{A}}_{k} \mathbf{e}_{k}+\sum_{i=2}^{k-1} h_{i}^{\alpha} \tilde{\mathbf{D}}_{k i} \mathbf{e}_{i}+\epsilon_{k}, \quad k=1,2, \ldots, N^{\prime} .
$$

Since both $\tilde{K}$ and $\tilde{M}$ are continuous functions on $I \times I$, by using Lemma 3.1 and (3.7) we observe that there exists a positive constant $c_{1}$ such that for $k=1,2, \ldots, N^{\prime}$ and $j, p=1,2, \ldots, r_{k}$

$$
\left|\tilde{a}_{j p}^{k}\right| \leq c_{1} \int_{0}^{\tau_{j}}\left[\left(\tau_{j}-\tau\right)^{\alpha-1}+1\right] d \tau \leq c_{1}\left(1+\frac{1}{\alpha}\right)
$$

and

$$
\left|\tilde{d}_{j p}^{k i}\right| \leq c_{1} \int_{0}^{1}\left(\frac{t_{k-1}-t_{i-1}+h_{k} \tau_{j}}{h_{i}}-\tau\right)^{\alpha-1} d \tau, \quad i=2,3, \ldots, k-1
$$

Estimate (4.14) implies that there exists a positive constant $c_{2}:=c_{1} \ell\left(1+\frac{1}{\alpha}\right)$ for all $k=1,2, \ldots, N^{\prime}$,

$$
\left\|\tilde{\mathbf{A}}_{k}\right\|_{\infty} \leq c_{2}
$$


Let $h:=\max \left\{h_{i}: 1 \leq i \leq N^{\prime}\right\}$ and choose $h<c_{2}^{-1 / \alpha}$. It follows that for such an $h$ the matrix $I-h_{k}^{\alpha} \tilde{\mathbf{A}}_{k}$ is invertible and there exists a positive constant $c$ such that

$$
\left\|\left(I-h_{k}^{\alpha} \tilde{\mathbf{A}}_{k}\right)^{-1}\right\| \leq c .
$$

Thus, from (4.13) we conclude that

$$
\mathbf{e}_{k}=\left(I-h_{k}^{\alpha} \tilde{\mathbf{A}}_{k}\right)^{-1}\left[\sum_{i=2}^{k-1} h_{i}^{\alpha} \tilde{\mathbf{D}}_{k i} \mathbf{e}_{i}+\epsilon_{k}\right] .
$$

We next use estimate (4.15) to study the bound of the entries of matrices $\tilde{\mathbf{D}}_{k i}$. Noting that $h_{i}<h_{i+1}<\cdots<h_{k-1}$, we have that

$$
\frac{t_{k-1}-t_{i-1}}{h_{i}}=\frac{h_{i}+\cdots+h_{k-1}}{h_{i}} \geq k-i
$$

and conclude that

$$
\left|\tilde{d}_{j p}^{k i}\right| \leq c_{1} \int_{0}^{1}(k-i-\tau)^{\alpha-1} d \tau .
$$

It can be verified from a direct computation that there exists a positive constant $c$ such that for all $i=2,3, \ldots, k-1$

$$
\int_{0}^{1}(k-i-\tau)^{\alpha-1} d \tau \leq c(k-i)^{\alpha-1}
$$

Using this estimate in inequality (4.18) yields for $i=2,3, \ldots, k-1$ and $j, p=$ $1,2, \ldots, r_{k}$ that

$$
\left|\tilde{d}_{j p}^{k i}\right| \leq c(k-i)^{\alpha-1}
$$

and thus

$$
\left\|\tilde{\mathbf{D}}_{k i}\right\|_{\infty} \leq c(k-i)^{\alpha-1} .
$$

Combining estimates (4.16) and (4.19) with (4.17) gives

$$
\left\|\mathbf{e}_{k}\right\|_{\infty} \leq c \sum_{i=2}^{k-1} h_{i}^{\alpha}(k-i)^{\alpha-1}\left\|\mathbf{e}_{i}\right\|_{\infty}+c\left\|\epsilon_{k}\right\|_{\infty} .
$$

Recalling from (3.10) that there exists a positive constant $c$ such that

$$
h_{i} \leq c\left(i+i_{0}-2\right)^{q-1} N^{-q},
$$

since $i+i_{0}-2 \leq 2 N$ we conclude that there exists a positive constant $c$ such that

$$
h_{i} \leq c N^{-1} \text {. }
$$

Therefore, we have that

$$
\left\|\mathbf{e}_{k}\right\|_{\infty} \leq c\left(\frac{1}{N}\right)^{\alpha} \sum_{i=2}^{k-1}(k-i)^{\alpha-1}\left\|\mathbf{e}_{i}\right\|_{\infty}+c\left\|\epsilon_{k}\right\|_{\infty} .
$$


We next use inequality (4.20) to obtain the error estimate of the hybrid collocation method (4.1). For this purpose, we recall a discrete Gronwall-type inequality (cf. [5]).

Lemma 4.1. Let $0<\alpha<1$ and $\left\{z_{i}: i=1,2, \ldots, n\right\}$ be a sequence of positive numbers and $n \leq N$. Let $\rho$ and $\beta$ be two positive numbers such that for all $k=$ $1,2, \ldots, n$

$$
z_{k} \leq\left(\frac{1}{N}\right)^{\alpha} \beta \sum_{i=1}^{k-1}(k-i)^{\alpha-1} z_{i}+\rho .
$$

Then there exists a positive constant $c$ depending only on $\alpha$ and $\beta$ such that $k=$ $1,2, \ldots, n$,

$$
z_{k} \leq c \rho .
$$

We are now ready to prove the main result of this paper, which gives an optimal order of global convergence for the hybrid collocation method.

THEOREM 4.2. Let $y$ be the exact solution of (1.1), let $N$ be a positive integer, and let $Q_{h}$ be the hybrid interpolation operator defined by (3.6) associated with the graded partition (3.4). Suppose that the forcing function $f$ in (1.1) has the form (2.1). Then, for sufficiently large $N,(4.1)$ has a unique solution $y_{h}$ and there exists a positive constant $c$ independent of $N$ such that

$$
\left\|y-y_{h}\right\| \leq c N^{-r} .
$$

Proof. It follows from Theorem 2.1 that the solution of (1.1) has the form $y=u+v$, where $u \in C^{r}(I)$ and $v \in V_{r}$. This allows us to use Proposition 3.4 to prove the result. We first estimate the error on $\left[t_{0}, t_{1}\right]$. From (4.3) there exists a constant $c$ such that

$$
\left\|\epsilon_{1}\right\|_{\infty} \leq\left\|T_{\alpha}\left(y-Q_{h} y\right)\right\|_{\left[t_{0}, t_{1}\right]} \leq c\left\|y-Q_{h} y\right\|_{\left[t_{0}, t_{1}\right]} .
$$

It follows from (4.20) and Proposition 3.4 that

$$
\left\|\mathbf{e}_{1}\right\|_{\infty} \leq c\left\|y-Q_{h} y\right\|_{\left[t_{0}, t_{1}\right]} \leq c N^{-r} .
$$

Using Lemma 3.1 and the above estimate we obtain that

$$
\left\|Q_{h}\left(y-y_{h}\right)\right\|_{\left[t_{0}, t_{1}\right]} \leq c\left\|\mathbf{e}_{1}\right\|_{\infty} \leq c N^{-r},
$$

which with Proposition 3.4 gives that

$$
\left\|y-y_{h}\right\|_{\left[t_{0}, t_{1}\right]} \leq\left\|Q_{h}\left(y-y_{h}\right)\right\|_{\left[t_{0}, t_{1}\right]}+\left\|Q_{h} y-y\right\|_{\left[t_{0}, t_{1}\right]} \leq c N^{-r} .
$$

Next we estimate the error on $\left[t_{1}, 1\right]$. Using (4.3) we have that for $k=2,3, \ldots, N^{\prime}$

$$
\left\|\epsilon_{k}\right\|_{\infty} \leq\left\|T_{\alpha, 1}\left(y-Q_{h} y\right)\right\|_{\left[t_{1}, 1\right]}+\left\|\left(T_{\alpha}-T_{\alpha, 1}\right)\left(y-y_{h}\right)\right\|_{\left[t_{1}, 1\right]} .
$$

Now, by using Proposition 3.4 and estimate (4.21) we conclude that there exists a positive constant $c$ such that

$$
\left\|\epsilon_{k}\right\|_{\infty} \leq c\left(\left\|y-Q_{h} y\right\|_{\left[t_{1}, 1\right]}+\left\|y-y_{h}\right\|_{\left[t_{0}, t_{1}\right]}\right) \leq c N^{-r} .
$$

Combining the above estimate with (4.20) we obtain that

$$
\left\|\mathbf{e}_{k}\right\|_{\infty} \leq c N^{-\alpha} \sum_{i=2}^{k-1}(k-i)^{\alpha-1}\left\|\mathbf{e}_{i}\right\|_{\infty}+c N^{-r} .
$$


Using Lemma 4.1 with $z_{1}=0$ and $z_{i}=\left\|\mathbf{e}_{i}\right\|_{\infty}$ for $i=2,3, \ldots N^{\prime}$, we conclude that there exists a positive constant $c$ such that for all $k=2,3, \ldots, N^{\prime}$

$$
\left\|\mathbf{e}_{k}\right\|_{\infty} \leq c N^{-r}
$$

Now, by the uniform boundedness (3.7) of $\left\|L_{i j}\right\|$ for $i=2,3, \ldots, N^{\prime}$ there exists a positive constant $c$ such that

$$
\left\|Q_{h}\left(y-y_{h}\right)\right\|_{\left[t_{1}, 1\right]} \leq c \max \left\{\left\|\mathbf{e}_{k}\right\|_{\infty}: k=2,3, \ldots, N^{\prime}\right\} \leq c N^{-r} .
$$

It follows that there exists a positive constant $c$ such that

$$
\left\|y-y_{h}\right\|_{\left[t_{1}, 1\right]} \leq\left\|Q_{h}\left(y-y_{h}\right)\right\|+\left\|Q_{h} y-y\right\| \leq c N^{-r},
$$

which concludes the proof of the theorem.

We may also use the compactness of operator $T_{\alpha}$ and the uniform boundedness of $Q_{h}$ to prove Theorem 4.2 (see, for example, [1]). In fact, such a proof is more concise. We choose the current proof, for it provides guidance for the construction of a numerical algorithm in our numerical experiments.

In the next proposition, we compare the graded collocation (GC) method with the hybrid collocation (HC) method. To this end, we let $\mathcal{N}_{G C}$ and $\mathcal{N}_{H C}$ denote the number of subintervals used in the GC method and the HC method, and we let $\mathcal{L}_{G C}$ and $\mathcal{L}_{H C}$ denote the length of the smallest subinterval used in the GC method and the HC method, respectively. We also consider the ratios of the largest subinterval over the smallest subinterval for the partitions that associate with the GC method and the HC method, which are denoted by $\mathcal{R}_{G C}$ and $\mathcal{R}_{H C}$. Such a ratio is a good measure for the stability of the corresponding collocation method.

Proposition 4.3. There hold the estimates that

$$
\begin{gathered}
\mathcal{N}_{G C}-\mathcal{N}_{H C} \geq \frac{N}{\sqrt[q]{N}}-1, \\
\mathcal{L}_{G C}=\frac{1}{N^{q}}, \quad \mathcal{L}_{H C} \geq \frac{\sqrt[q]{N}}{N^{2}},
\end{gathered}
$$

and

$$
\mathcal{R}_{G C} \geq q(N-1)^{q-1}, \quad \mathcal{R}_{H C} \leq \frac{q N}{\sqrt[q]{N}}
$$

Proof. Since $\mathcal{N}_{G C}=N$ and $\mathcal{N}_{H C}=N-i_{0}+1 \leq N-N^{1-1 / q}+1$, we have that

$$
\mathcal{N}_{G C}-\mathcal{N}_{H C} \geq N-\left(N-N^{1-1 / q}+1\right)=\frac{N}{\sqrt[q]{N}}-1 .
$$

The smallest interval used in the GC method is $\left[0, N^{-q}\right]$ and thus $\mathcal{L}_{G C}=\frac{1}{N^{q}}$. For the HC method, it is easily verified that $\mathcal{L}_{H C}=\min \left\{h_{1}, h_{2}\right\}$, where $h_{1}=\left(\frac{i_{0}}{N}\right)^{q}$ and $h_{2}=\left(\frac{i_{0}+1}{N}\right)^{q}-\left(\frac{i_{0}}{N}\right)^{q}$. Recalling that $i_{0}$ satisfies that condition $N^{1-\frac{1}{q}} \leq i_{0} \leq$ $N(N-1)^{-\frac{1}{q}}$, we derive that

$$
h_{1} \geq\left(\frac{N^{1-\frac{1}{q}}}{N}\right)^{q}=\frac{1}{N} \geq \frac{\sqrt[q]{N}}{N^{2}} .
$$


On the other hand, there exists a constant $\theta$ with $0<\theta<1 / i_{0}$ such that

$$
h_{2}=q i_{0}^{q-1}(1+\theta)^{q-1} N^{-q} .
$$

Thus

$$
h_{2} \geq q i_{0}^{q-1} N^{-q} \geq q N^{\frac{1}{q}-2} \geq \frac{\sqrt[q]{N}}{N^{2}} .
$$

This concludes the second estimate in this proposition. The third estimate can be similarly obtained.

We remark that it follows from Proposition 4.3 that the $\mathrm{HC}$ method requires less computational cost than the GC method even though they have the same order of convergence. For example, when $\alpha=\frac{1}{2}, r=3$, and $N=100$, the HC method uses 54 subintervals while the GC method uses 100 subintervals.

Another important point made in the last proposition is that the $\mathrm{HC}$ method is more stable than the GC method since the length of the smallest subinterval used in the HC method is larger than the length of the smallest subinterval used in the GC method. Notice that the length of the smallest interval used in the HC method is not as sensitive to $r$ and $\alpha$ as that in the GC method. For instance, when $N=1000, \alpha=$ $\frac{1}{2}$, and $r=3$, the length of the smallest interval used in the GC method is $h_{1}=10^{-24}$ while the length of the smallest interval used the $\mathrm{HC}$ method is $4.217 \times 10^{-5}$. In addition, we see from the proposition that for the GC method $\mathcal{R}_{G C}$ grows in the order $\mathcal{O}\left(N^{q-1}\right)$ while for the HC method $\mathcal{R}_{H C}$ grows slower than $\mathcal{O}(N)$. When $p$ is large, which is the case when $\alpha$ is small or $r$ is large, $\mathcal{R}_{G C}$ is extremely large. This may cause serious instability problems. The result in the proposition shows that the HC method is much more stable than the GC method.

5. Numerical experiments. In this section, we report results of numerical experiments which confirm the theoretical analysis for the HC method presented in the last section and demonstrate the effectiveness of the method.

In (1.1) we choose $K(s, t)=M(s, t)=1, \alpha=1 / 2$ and choose $f$ such that the equation has the exact solution

$$
y(t)=\sqrt{t^{2}+t} \cos t+\sin t, \quad t \in I .
$$

Note that the first derivative of this solution has a singularity at $t=0$.

The purpose of these numerical experiments is to compare the numerical performance of the HC method with the GC method. For both of the methods we use piecewise polynomials of degree 2 , that is, $r=3$, and, in addition, for the HC method we use

$$
V_{3}=\operatorname{span}\left\{1, t, t^{2}, t^{\frac{1}{2}}, t^{\frac{3}{2}}, t^{\frac{5}{2}}\right\}
$$

on the first interval.

Tables 5.1 and 5.2 are given to compare the numerical performance of the two methods, where "order of conv." stands for the order of convergence. The weakly singular integrals that appear in these methods are computed by a numerical integration scheme presented in [12] specifically designed for weakly singular integrals of this type.

The HC method and the GC method have the same orders of convergence. The computed orders of convergence are consistent with the theoretical order, which is $r=$ 
TABLE 5.1

Numerical performance of the $H C$ method.

\begin{tabular}{|c|c|c|c|c|}
\hline$N$ & $\left\|y-y_{h}\right\|$ & Order of conv. & $\mathcal{N}_{H C}$ & $\mathcal{L}_{H C}$ \\
\hline 20 & $3.666 \mathrm{e}-4$ & - & 8 & $7.5419 \mathrm{e}-02$ \\
40 & $4.680 \mathrm{e}-5$ & 2.9698 & 19 & $2.7681 \mathrm{e}-02$ \\
60 & $1.408 \mathrm{e}-5$ & 2.9616 & 30 & $1.9022 \mathrm{e}-02$ \\
80 & $5.983 \mathrm{e}-6$ & 2.9759 & 42 & $1.3423 \mathrm{e}-02$ \\
100 & $3.069 \mathrm{e}-6$ & 2.9916 & 54 & $1.0779 \mathrm{e}-02$ \\
\hline
\end{tabular}

TABLE 5.2

Numerical performance of the GC method.

\begin{tabular}{|c|c|c|c|c|}
\hline$N$ & $\left\|y-y_{h}\right\|$ & Order of conv. & $\mathcal{N}_{G C}$ & $\mathcal{L}_{G C}$ \\
\hline 20 & $1.993 \mathrm{e}-4$ & - & 20 & $1.5625 \mathrm{e}-08$ \\
40 & $3.446 \mathrm{e}-5$ & 2.9720 & 40 & $2.4414 \mathrm{e}-10$ \\
60 & $1.240 \mathrm{e}-5$ & 2.9576 & 60 & $2.1433 \mathrm{e}-11$ \\
80 & $6.011 \mathrm{e}-6$ & 2.9776 & 80 & $3.8147 \mathrm{e}-12$ \\
100 & $3.430 \mathrm{e}-6$ & 2.9896 & 100 & $1.0000 \mathrm{e}-12$ \\
\hline
\end{tabular}

3 for both methods. In terms of convergence both methods give satisfactory numerical performance. However, the HC method uses much fewer subintervals. Therefore, it requires less computational cost than the $\mathrm{GC}$ method. Also, the length of the smallest subinterval used in the $\mathrm{HC}$ method is significantly larger than that in the GC method. When $N$ is large and $\alpha$ is small, for the GC method, the length of the first interval is extremely small, which may cause serious round-off errors. The HC method has a rather uniform partition, which avoids the problem of having small subintervals. In these two aspects, the HC method has performed better than the GC method.

Acknowledgment. The authors are grateful to two referees for their suggestions that improve the presentation of this paper.

\section{REFERENCES}

[1] K. E. Atkinson, The Numerical Solution of Integral Equations of the Second Kind, Cambridge University Press, Cambridge, UK, 1997.

[2] H. BRUnNer, Nonpolynomial spline collocation for Volterra equations with weakly singular kernels, SIAM J. Numer. Anal., 20 (1983), pp. 1106-1119.

[3] H. Brunner, The numerical solution of weakly singular Volterra integral equations by collocation on graded meshes, Math. Comp., 45 (1985), pp 417-437.

[4] H. BRunner, The numerical solution of weakly singular first-kind Volterra integral equations with delay arguments, Proc. Estonian Acad. Sci. Phys. Math., 48 (1999), pp. 90-100.

[5] H. Brunner and P. J. van der Houwen, The Numerical Solution of Volterra Equations, North-Holland, Amsterdam, 1986.

[6] H. Brunner, A. Pedas, and G. Vainikko, The piecewise polynomial collocation method for nonlinear weakly singular Volterra equations, Math. Comp., 68 (1999), pp. 1079-1095.

[7] J. Burns, E. Cliff, and T. Herdman, A state-space model for an aeroelastic system, in Proceedings of the 22nd IEEE Conference on Decision and Control, San Antonio, TX, 1983, pp. 1174-1177.

[8] Y. CaO AND Y. Xu, Singularity preserving Galerkin methods for weakly singular Fredholm integral equations, J. Integral Equations Appl., 6 (1994), pp. 303-334.

[9] T. Diogo, S. McKee, And T. TANG, A Hermite-type collocation method for the solution of integral equations with a certain weakly singular kernels, IMA J. Numer. Anal., 11 (1991), pp. 595-605.

[10] T. Diogo, S. McKee, And T. TAng, Collocation methods for second-kind Volterra integral equations with weakly singular kernels, Proc. Royal Soc. Edinburgh, 124 (1994), pp. 199210. 
[11] B. Jumarhon, W. Lamb, S. McKee, and T. Tang, A Volterra integral type method for solving a class of nonlinear initial-boundary value problems, Numer. Methods Partial Differential Equations, 12 (1996), pp. 265-281.

[12] H. KANEKO AND Y. XU, Gaussian-type quadratures for weakly singular integrals and their applications to the Fredholm integral equation of the second kind, Math. Comp., 62 (1994), pp. 739-753.

[13] J. RICE, On the degree of convergence of nonlinear spline approximations, in Approximation with Special Emphasis on Spline Functions, I. J. Schoenberg, ed., Academic Press, NY, 1969, pp. 349-365.

[14] H. J. RIELE, Collocation methods for weakly singular second-kind Volterra integral equations with non-smooth solution, IMA J. Numer. Anal., 2 (1982), pp. 437-449.

[15] T. TANG, Superconvergence of numerical solutions to weakly singular Volterra integraldifferential equations, Numer. Math., 61 (1992), pp. 373-382.

[16] T. TANG, A note on collocation methods for Volterra integro-differential equations with weakly singular kernels, IMA J. Numer. Anal., 13 (1993), pp. 93-99. 